\title{
Gambling for resurrection and the heat equation on a triangle
}

\author{
Stefan Ankirchner * Christophette Blanchet-Scalliet ${ }^{\dagger}$ \\ Nabil Kazi-Tani $\ddagger \quad$ Chao Zhou $\S$
}

December 11, 2019

We consider the problem of controlling the diffusion coefficient of a diffusion with constant negative drift rate such that the probability of hitting a given lower barrier up to some finite time horizon is minimized. We assume that the diffusion rate can be chosen in a progressively measurable way with values in the interval $[0,1]$. We prove that the value function is regular, concave in the space variable, and that it solves the associated HJB equation. To do so, we show that the heat equation on a right triangle, with a boundary condition that is discontinuous in the corner, possesses a smooth solution.

$2010 M S C:$ 60G44, 49L20, 35C10.

Keywords : Hitting probability, stochastic control, heat equation.

\section{Introduction}

Let $\left(\Omega, \mathcal{F},\left(\mathcal{F}_{t}\right)_{t \geqslant 0}, P\right)$ be a filtered probability space supporting a standard onedimensional Brownian motion $W$. Let $T \in(0, \infty)$ be a finite time horizon. We denote by $\mathcal{A}$ the set of all $\left(\mathcal{F}_{t}\right)$-progressively measurable stochastic processes $\left(\alpha_{t}\right)_{t \in[0, T]}$ with values in $[0,1]$. For every $\alpha \in \mathcal{A}$ and $(t, x) \in[0, T] \times \mathbb{R}$ we define the stochastic process

$$
X_{s}^{t, x, \alpha}=x-(s-t)+\int_{t}^{s} \gamma \alpha_{r} d W_{r}, \quad s \in[t, T]
$$

where $\gamma$ is a positive constant.

For all $(t, x) \in[0, T] \times[0, \infty)$ and $\alpha \in \mathcal{A}$ we define the cost functional

$$
J(t, x, \alpha)=P\left(\inf _{t \leqslant s \leqslant T} X_{s}^{t, x, \alpha}<0\right) .
$$

${ }^{*}$ Stefan Ankirchner, Institute for Mathematics, University of Jena, Ernst-Abbe-Platz 2, 07743 Jena, Germany. Email: s.ankirchner@uni-jena.de, Phone: +49 (0)3641 946275.

${ }^{\dagger}$ Christophette Blanchet-Scalliet, Université de Lyon - CNRS, UMR 5208, Institut Camille Jordan - Ecole Centrale de Lyon, 36 avenue Guy de Collongue, 69134 Ecully Cedex, FRANCE. Email: christophette.blanchet@ec-lyon.fr, Phone: +33472186405

${ }^{\ddagger}$ Nabil Kazi-Tani, ISFA, Université de Lyon 1, 50 Avenue Tony Garnier, 69007 Lyon, France Email: nabil.kazitani@univ-lyon1.fr, Phone: +33 (0)437287474.

$\S$ Department of Mathematics, National University of Singapore, Singapore, matzc@nus.edu.sg. Research supported by NUS Grant R-146-000-179-133. 
In this article we consider the control problem that consists in minimizing the probability (1.2). To this end we introduce the value function

$$
V(t, x)=\inf \{J(t, x, \alpha): \alpha \in \mathcal{A}\}
$$

for all $(t, x) \in[0, T] \times[0, \infty)$.

The control problem is rather generic and allows for various interpretations. For example one can interpret the state process (1.1) as the value process of a company that is going through a phase where its obligations exceed its average revenues (e.g. due to a recession or a market change). The problem of minimizing (1.2) describes in a stylized form the situation of a manager aiming at minimizing the company's bankruptcy probability up to time $T$. The manager can choose between some riskier and less risky strategies, and thus determine the volatility of the company value process. Here, $T$ can be interpreted, e.g., as the end of the phase with a negative trend or the end of the manager's employment period. A more specific interpretation within an insurance application is given below.

Alternatively, one can interpret the state process (1.1) as an animal's level of energy reverves during a period with little food, e.g. the winter. The animal can choose among various food searching strategies. Some strategies increase the probability of finding food, but also require a higher energy consumption. The problem of minimizing (1.2) then describes the animal's aim of minimizing the death probability until the end of the winter. Let us now briefly indicate our general strategy and tools to solve the considered stochastic control problem.

Summary of the approach Suppose that $x \geqslant T$ at time zero. By choosing the control $\alpha=0$, the state process $X$ is greater than or equal to zero up to time $T$. Therefore, $V(0, x)=0$ for all $x \geqslant T$. More generally, $V(t, x)=0$ for all $x \geqslant T-t$. In particular, as soon as the state process is greater than or equal to time to maturity, then it is optimal to choose the control $\alpha=0$ until maturity $T$.

Additionally, we prove in this paper that whenever the state process is smaller than time to maturity, it is optimal to set the control $\alpha$ equal to one. In other words, an optimal control can be described in terms of the feedback function $a^{\star}$ : $[0, T] \times \mathbb{R} \rightarrow[0,1]$, defined by

$$
a^{\star}(t, x)= \begin{cases}1, & \text { if } x<T-t, \\ 0, & \text { otherwise. }\end{cases}
$$

We can link the value function to the heat equation on a right triangle. To explain this, let $Y^{t, x}$ be the state process controlled with feedback function $a^{\star}$. Then the value function at a point $(t, x)$ with $0 \leqslant x<T-t$ is equal to the probability for $Y^{t, x}$ to attain zero before hitting the line $T-s, s \geqslant t$. Notice that the process $Y^{t, x}$ is a Brownian motion with drift -1 and with constant volatility $\gamma$, until it hits the line $T-s, s \geqslant t$ for the first time. Thus, for $t \in[0, T)$ and $x \in(0, T-t)$, we can prove that the value function coincides with the probability for $Y^{t, x}$ to first exit the right triangle with vertices $(t, 0),(t, T-t)$ and $(T, 0)$ from the opposite side of the hypotenuse (called cathetus), as illustrated in Figure 1a (where $\sigma^{t, x}:=\inf \{s \geqslant t$ : $Y_{s}^{t, x} \geqslant T-s$ or $\left.\left.Y_{s}^{t, x} \leqslant 0\right\}\right)$.

The exit probability for $Y^{t, x}$ can be reduced to an exit probability for a Brownian motion, which will provide the link with the heat equation. To give a precise 


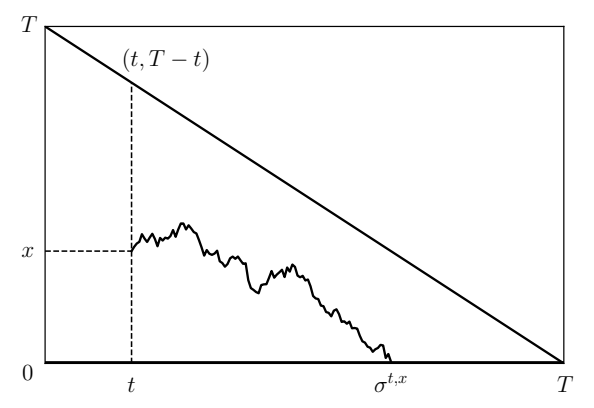

(a) $Y^{t, x}$ exits the triangle from the cathetus.

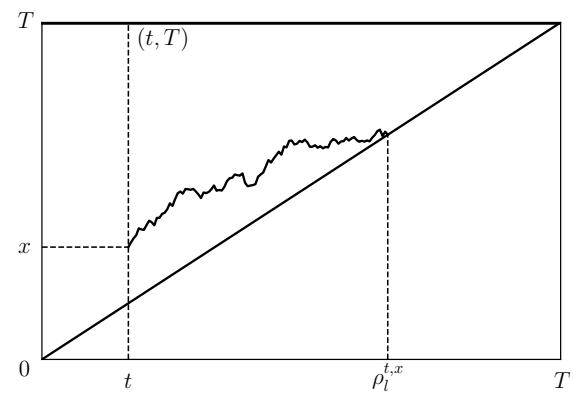

(b) First exit time of $\gamma W^{t, x}$ from the triangle $\Delta$.

statement we define, for all $t \in[0, T)$ and $x \in(t, T)$

$$
\begin{gathered}
\rho_{u}^{t, x}=\inf \left\{s \geqslant t: \gamma W_{s}^{t, x} \geqslant T\right\}, \\
\rho_{l}^{t, x}=\inf \left\{s \geqslant t: \gamma W_{s}^{t, x} \leqslant s\right\},
\end{gathered}
$$

where $\left(W_{s}^{t, x}\right)_{s \geqslant t}$ is a Brownian motion starting in $x$ at time $t$. Let $\Delta$ be the right triangle with vertices $(t, t),(t, T)$ and $(T, T)$, and define the following quantity

$$
H(t, x):=P\left(\rho_{l}^{t, x}<\rho_{u}^{t, x}\right),
$$

which is the probability that $\gamma W^{t, x}$ exits $\Delta$ from the hypothenuse (see Figure 1b).

In this article, we derive an explicit formula for the function $H$, using known exit probabilities for a Bessel bridge from a wedge [22]. Moreover, we show that $H$ is a classical solution of the heat equation

$$
\partial_{t} H(t, x)+\frac{\gamma^{2}}{2} \partial_{x x} H(t, x)=0, \quad 0 \leqslant t<T, t<x<T,
$$

with the boundary conditions

$$
\begin{gathered}
H(t, T)=0, \text { for all } t \in[0, T], \\
H(t, t)=1, \text { for all } t \in[0, T) .
\end{gathered}
$$

Note the irregularity of the conditions at the triangle vertex $(T, T)$, which is excluded. With the smoothness properties of $H$, we can perform a verification and show that the value function of the control problem (1.3) satisfies

$$
V(t, x)=H(t, x+t), \quad t \in[0, T], x \in[0, t],
$$

and conclude that $a^{\star}$ is indeed an optimal control.

Related literature The problem under study here is related to several subjects: ruin probability in insurance, control of occupation times, volatility uncertainty in finance, or the heat equation on a triangle. Let us briefly discuss the related literature on each of these aspects.

A similar version of the control problem considered in this article has been studied in the literature on reinsurance optimization. To explain this, we remark that one can interpret $X$ as the diffusion approximation of the surplus process of an insurance company and $\alpha_{t}$ as the retention rate at time $t$, i.e. fraction of the instantaneous 
claims rate paid by the insurance company. In this interpretation the fraction $\left(1-\alpha_{t}\right)$ of the claims is transferred to a reinsurance company without any additional costs.

Minimizing (1.2) then corresponds to minimizing, up to time $T$, the so-called ruin probability, the central quantity for measuring risk within risk theory (see e.g. [4]). When ultimate ruin probability is used as a criteria to be minimized, several results are known: Schmidli [23] studies both cases of diffusion approximations and classical risk model, and shows in particular that the value function is concave, using dynamic programming arguments. An extension to a model where investments are also allowed can be found in $[25,13]$. If Excess-of-Loss reinsurance is used instead of linear contracts, a verification theorem is provided in [16]. An argument of different nature has been described in [8], which allows to minimize hitting probabilities for diffusions, while controlling the drift and volatility terms, and which uses stochastic orders in place of the usual dynamic programming tools. Note that this argument cannot be used in our model, since we allow zero as a possible value for the volatility. Criteria which are different from ruin probability have also been considered: since the ruin probability can be hard to compute, Liang and Guo [17] considered maximizing the adjustment coefficient, which appears as an upper bound for the ruin probability in Lundberg's inequality. Browne [12] has given conditions, in an optimal investment setting, under which ruin probability minimization is equivalent to exponential utility maximization. Again, the literature on optimal control in insurance mathematics is large, and we refer the reader to the monographs $[24,4,7]$ for more details and references.

In all the papers of the literature mentioned above, only the infinite time ruin probability has been considered for reinsurance optimization. In that case, the HJB equation for the value function is an ODE. One consequence of the introduction of a finite maturity time is that the HJB equation becomes a PDE, whose regularity is in general much more difficult to verify. In this paper, we are able to prove the regularity of the value function directly, using an explicit expression, derived using the distribution of particular Bessel bridges (see Section 3). This is the second main contribution of this paper: the computation of an explicit solution of the heat equation on a triangle, with discontinuous boundary conditions.

Minimizing (1.2), the bankruptcy/ruin/death probability up to time $T$, is related to the problem of maximizing the time spent positive by a Brownian diffusion. Optimizing occupation times of continuous diffusions has received some attention in the literature. McNamara [19] considers the control of the volatility coefficient of a Brownian martingale, determines necessary and sufficient conditions on the objective functional such that the optimal control is bang-bang: the diffusion coefficient can vary between two positive real values $\sigma_{1}$ and $\sigma_{2}$ and the optimal control only takes these two extremal values. In a discrete time and discrete state space setting, Althöfer et al. [2] analyze the case where the controller can choose the step size of a discrete random walk, in order to maintain it in a given region, and also obtain bang-bang optimal controls. A related occupation time control problem for exponential martingales in continuous time is solved in [3]. Volatility control with bang-bang solutions appears in various fields of applications such as ecology [27], where the context is the minimization of a starvation probability for animals, or insurance mathematics [5], in the case of an optimal dividend problem.

Optimization problems over a set of volatility processes taking values in a bounded interval also arise in mathematical finance within models with volatility uncertainty. In that particular context, it is assumed that the volatility coefficient is uncertain, 
lying between two deterministic bounds. A model for pricing and hedging of options under volatility uncertainty is presented in [6], analyzing no arbitrage price bounds, that arise as solutions of a non linear partial differential equation (PDE), called the Black-Scholes-Barenblatt equation by the authors, and which is of the form of the HJB equation (4.2) associated to our control problem. Again, the result is bang-bang: the volatility selected for pricing only takes the two extreme values. This model has been extended in [14], where the uncertainty is specified by a family of martingale measures. The model uncertainty point of view described in these papers corresponds to a stochastic control problem, since the reasoning adopted is the selection of the worse case scenario: not knowing the volatility consists then in selecting the most unfavourable volatility. Peng [20] provides a framework for volatility uncertainty analysis, where the starting point is not a usual probability space, but a PDE, which is also analogous to (4.2). The literature around volatility uncertainty in mathematical finance is abundant, and the very brief overview that we give here is by no means exhaustive. In particular, several authors deal with the problem of robust utility maximization, which is usually formulated as a control problem under model uncertainty. When the volatility is uncertain, several examples of bang-bang and non bang-bang explicit solutions are analyzed in [18].

As mentioned above, in this paper, we reduce the analysis of the value function to the study of the heat equation in a right triangle, using Brownian motion on the real line. If a two dimensional Brownian motion is considered, the formulas are completely different, and explicit expressions are obtained for exit probabilities from a right-angled isoceles triangle in [26], using a conformal application, that transforms the triangle into a strip. In the case of an equilateral triangle and Dirichlet boundary conditions, a solution is given in [15], that depends on an integral transform of the boundary condition.

The rest of the paper is organised as follows: In Section 2, we precisely define the stochastic control problem and provide a solution, together with some economic interpretation. Section 3 is devoted to the explicit solution of the heat equation on a triangle and in Section 4, we solve the control problem.

\section{The control problem: analytic solution and economic interpretation}

Let us first introduce more precisely the stochastic basis that we will consider. Let $\Omega=\mathcal{C}([0, \infty), \mathbb{R})$ be the space of all continuous real valued functions and $\left(W_{t}\right)_{t \geqslant 0}$ be the coordinate process, i.e. $W_{t}(\omega)=\omega(t), t \geqslant 0, \omega \in \Omega$. Let $\left(\mathcal{F}_{t}^{W}\right)_{t \geqslant 0}$ be the filtration generated by $\left(W_{t}\right)_{t \geqslant 0}$ and $\mathcal{F}^{W}:=\bigvee_{t \geqslant 0} \mathcal{F}_{t}^{W}$. Processes are identified in $\left(\Omega, \mathcal{F}^{W}\right)$ via their probability measures: in particular, $P_{x}$ denotes the probability under which the coordinate process is a Brownian motion with $P_{x}\left(W_{0}=x\right)=1$. Set $\mathcal{F}_{t}^{x}=\sigma\left(\mathcal{F}_{t}^{W}, \mathcal{N}^{x}\right), t \geqslant 0$, where $\mathcal{N}^{x}$ denotes the collection of $P_{x}$-null sets in $\mathcal{F}^{W}$. We set $\mathcal{F}_{t}=\bigcap_{x} \mathcal{F}_{t}^{x}$ and $\mathcal{F}=\bigcap_{x} \mathcal{F}^{x}$.

We denote by $\mathcal{A}$ the set of all $\left(\mathcal{F}_{t}\right)$-progressively measurable stochastic processes $\left(\alpha_{t}\right)_{t \in[0, T]}$ with values in $[0,1]$. For every $\alpha \in \mathcal{A}$ and $(t, x) \in[0, T] \times \mathbb{R}$ we define the stochastic process

$$
X_{s}^{t, x, \alpha}=x-(s-t)+\int_{t}^{s} \gamma \alpha_{r} d W_{r}, \quad s \in[t, T], P-\text { a.s. }
$$


where $\gamma>0$ and $P:=P_{0}$.

We can introduce now the value function of the control problem that consists in minimizing a finite time ruin probability:

$$
V(t, x)=\inf \left\{P\left(\inf _{t \leqslant s \leqslant T} X_{s}^{t, x, \alpha}<0\right): \alpha \in \mathcal{A}\right\},
$$

for all $(t, x) \in[0, T] \times[0, \infty)$.

The following Theorem is the main result of this paper, and provides an analytic formula of the value function $V(t, x)$ as well as an optimal control.

Theorem 2.1. The value function satisfies, for all $(t, x) \in[0, T] \times(0, \infty)$ with $x<T-t$,

$$
V(t, x)=1-\int_{0}^{T-t} f\left(\frac{T-t-x}{\gamma}, v\right) g\left(\frac{T-t-x}{\gamma}, v, \frac{T-t-v}{\gamma}, \frac{1}{\gamma}\right) d v
$$

where

$$
f(z, v)=\frac{|z|}{\sqrt{2 \pi} v^{3 / 2}} \exp \left(-\frac{z^{2}}{2 v}\right), \quad(z, v) \in \mathbb{R} \times(0, \infty)
$$

and

$$
g(y, u, a, b)=\sum_{j=-\infty}^{\infty}\left(1-2 j \frac{a+u b}{y}\right) e^{-\frac{2 a}{u} j^{2}(a+u b)} e^{\frac{2 a}{u} j y}
$$

for all $a, b, u \in(0, \infty)$ and $y \in(0, a+b u)$. Moreover, for $(t, x) \in[0, T] \times[0, \infty)$ the process $a^{\star}\left(s, Y_{s}^{t, x}\right)_{s \in[t, T]}$ is an optimal control for $V(t, x)$, where $Y^{t, x}$ is the solution of the $S D E d Y_{s}=-d s+a^{\star}\left(s, Y_{s}\right) \gamma d W_{s}$ on $[t, T]$ with initial condition $x$, and where $a^{\star}$ is defined in (1.4).

The proof of Theorem 2.1 is postponed to Section 4. In the remainder of the current section we give an economic interpretation of the results of Theorem 2.1.

We refer to the region where time to maturity exceeds the state value as the losing region, and the complement as the winning region. Notice that $(t, x)$ is in the losing region if and only if $E^{P}\left(X_{T}^{t, x, \alpha}\right)<0$ for all $\alpha$. Theorem 2.1 states that, as long as the state process is in the losing region, it is optimal for the controller to choose $\alpha=1$. By choosing $\alpha=1$, the volatility of the state process is maximized. This further increases the probability for the state process to return to the winning region before $T$.

The controller only cares about ending up in the winning region at time $T$. In case of a ruin (or death) it does not matter for him by how far the state process $X$ dives into the negative area. In other words, the controller is "gambling for resurrection" in the losing region.

We now consider a more specific interpretation where the controller is a manager of a company aiming at minimizing the ruin probability up to time $T$. The strategy $a^{*}$ is optimal from the manager's perspective, but may not be so from a principal's perspective, e.g. a regulator or a company owner. Indeed, in choosing the strategy $a^{\star}$ the manager only cares about the probability of staying above the ruin level, but he does not care about the size of the loss, in the event where ruin occurs. A principal may care about the size of the conditional loss. In this sense, Theorem 2.1 shows that the ruin probability, as a target functional, can give wrong incentives when determining optimal management strategies. 


\section{The probability for a Brownian motion to exit a right triangle from the hypotenuse}

In this section we consider the probability for a one-dimensional Brownian motion to first exit the right triangle $\Delta_{b}$ in $\mathbb{R}^{2}$ with vertices $(t, b t),(t, b T)$ and $(T, b T)$ from its hypotenuse, where $b, T>0$. Note that we have to introduce a new parameter $b$ to properly take into account the parameter $\gamma$ appearing in (1.5) or (1.6)

Let $J=\{(t, x) \in[0, T) \times(0, b T): x \geqslant t\}$ and $\bar{J}=\{(t, x) \in[0, T] \times[0, b T]: x \geqslant t\}$. Let $(t, x) \in \bar{J}$ and $W^{t, x}$ a Brownian motion starting in $x$ at time $t$. We define

$$
\begin{aligned}
& \rho_{u}^{t, x}=\inf \left\{s \geqslant t: W_{s}^{t, x} \geqslant b T\right\} \\
& \rho_{l}^{t, x}=\inf \left\{s \geqslant t: W_{s}^{t, x} \leqslant b s\right\} .
\end{aligned}
$$

and

$$
H(t, x):=P\left(\rho_{l}^{t, x}<\rho_{u}^{t, x}\right) .
$$

The analytic formula that we derive for $H$ takes the following form.

Proposition 3.1. For all $(t, x) \in J$ we have

$$
H(t, x)=1-\int_{0}^{T-t} f(b T-x, v) g(b T-x, v, b(T-t-v), b) d v
$$

where the functions $f$ and $g$ are defined as in (2.2) and (2.3), respectively.

Let us comment on the methodology used to obtain (3.2), which also explains the origin of the functions $f$ and $g$. The integral term in the right hand side of (3.2) is the probability that $W^{t, x}$ exits $\Delta_{b}$ from the opposite side of the hypothenuse. The strategy that we use to compute this probability is to first fix the instant of time at which $W^{t, x}$ hits the horizontal boundary $y=b T$. In doing so, the process distribution obtained by conditionning the Brownian trajectory to first hit the level $b T$ at a deterministic time, say $u \in(t, T)$, is the bridge of a 3-dimensional Bessel process (see [10,9]), which is represented in Figure 2. In [9], this process is called the Brownian first passage bridge, and other representations and transformations of this process are given, including in the discrete time random walk case. Now, what we want is that this process stays above the hypothenuse during its whole lifetime interval $u-t$ : Lemma 3.2 below says that this probability is given by the function $g$. It remains to multiply by the first hitting time density $f$ of level $b T$ of $W^{t, x}$, evaluated at $u$, and to integrate for all values of $u$ in $(t, T)$. Said otherwise, we will use that

$$
1-H(t, x)=\int_{t}^{T} P_{0, u-t, b T-x}^{B E S}\left(W_{s}<b(s+T-u), s \in(0, u-t)\right) f(u) d u,
$$

where $P_{x, u, y}^{B E S}$ denotes the law on $\Omega$ of the 3 -dimensional Bessel bridge between $x$ and $y$, with length $u>0$ and where $f$ is the density of the first hitting time of the level $b T-x$ by a standard Brownian motion. After a simple change of variable, this explains the formula appearing in (3.2).

For the proof of Proposition 3.1 we use the following lemma.

Lemma 3.2. Let $a>0, b>0, u>0$ and $y \in(0, a+b u)$. Then, by denoting $P_{x, u, y}$ the law of a Brownian bridge from $x$ to $y$ with length $u$, we have

$$
P_{0, u, y}\left(W_{s}<a+b s \text { for all } s \in[0, u] \mid W_{s}>0 \text { for all } s \in(0, u]\right)=g(y, u, a, b) .
$$




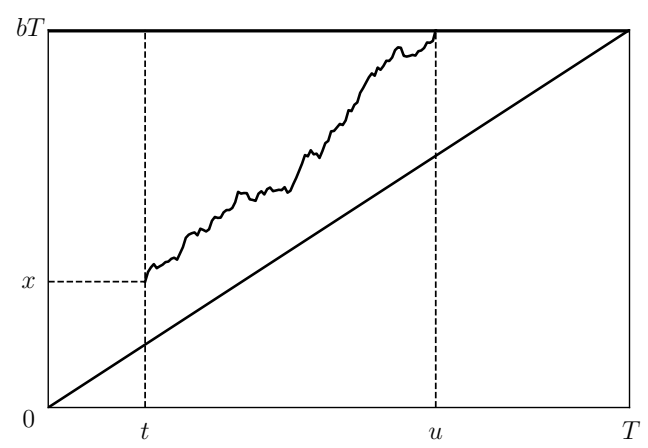

Figure 2: Hitting time conditioning and 3-dimensional Bessel bridge

Proof. For the special case $b=1$ the formula appears as formula (38) in [22]. For the reader's convencience we provide a sketch of the proof. It is known that $P_{x, u, y}\left(W_{s}>0\right.$ for all $\left.s \in(0, u]\right)=1-e^{-2 x y / u}$ (see e.g. page 174 in [11]). We next recall a formula for the probability that a Brownian bridge crosses a given line (see Theorem 3.3 and Prop 3.5 in [1] or Theorem 7 in [22]). We have

$$
\begin{aligned}
& P_{x, u, y}\left(0<W_{s}<a+b s \text { for all } s \in(0, u)\right) \\
& =P_{0, u, y-x}\left(-x<W_{s}<a-x+b s \text { for all } s \in(0, u)\right) \\
& =1-\sum_{k=1}^{\infty}\left(e^{-2 A_{k}(x)}+e^{-2 B_{k}(x)}-e^{-2 C_{k}(x)}-e^{-2 D_{k}(x)}\right),
\end{aligned}
$$

where

$$
\begin{aligned}
& A_{k}(x)=\frac{1}{u}\left(j^{2}(a-x)(a-y+b u)+(j-1)^{2} x y+j(j-1)[(a-y+b u) x+(a-x) y]\right), \\
& B_{k}(x)=\frac{1}{u}\left((j-1)^{2}(a-x)(a-y+b u)+j^{2} x y+j(j-1)[(a-y+b u) x+(a-x) y]\right), \\
& C_{k}(x)=\frac{1}{u}\left(j^{2}[(a-x)(a-y+b u)+x y]+j(j-1)(a-y+b u) x+j(j+1)(a-x) y\right), \\
& D_{k}(x)=\frac{1}{u}\left(j^{2}[(a-x)(a-y+b u)+x y]+j(j+1)(a-y+b u) x+j(j-1)(a-x) y\right) .
\end{aligned}
$$

A straightforward calculation shows

$$
\begin{array}{r}
A_{k}^{\prime}(x)=\frac{1}{u}(y-j(a+b u)), \\
\left.B_{k}^{\prime}(x)=\frac{1}{u}((j-1)(a+b u)+y)\right), \\
C_{k}^{\prime}(x)=-\frac{1}{u} j(a+b u), \\
D_{k}^{\prime}(x)=\frac{1}{u} j(a+b u) .
\end{array}
$$


With L'Hopital's rule we obtain

$$
\begin{aligned}
& \lim _{x \downarrow 0} \frac{P_{x, u, y}\left(W_{s}<a+b s \text { for all } s \in[0, u]\right)}{P_{x, u, y}\left(W_{s}>0 \text { for all } s \in(0, u]\right)} \\
& =\lim _{x \downarrow 0} \frac{\frac{\partial}{\partial x}\left[1-\sum_{k=1}^{\infty}\left(e^{-2 A_{k}(x)}+e^{-2 B_{k}(x)}-e^{-2 C_{k}(x)}-e^{-2 D_{k}(x)}\right)\right]}{\frac{\partial}{\partial x}\left[1-e^{-2 x y / u}\right]} \\
& =\frac{u}{y} \sum_{k=1}^{\infty}\left(A_{k}^{\prime}(0) e^{-2 A_{k}(0)}+B_{k}^{\prime}(0) e^{-2 B_{k}(0)}-C_{k}^{\prime}(0) e^{-2 C_{k}(0)}-D_{k}^{\prime}(0) e^{-2 D_{k}(0)}\right) \\
& =\sum_{j=-\infty}^{\infty}\left(1-2 j \frac{a+u b}{y}\right) e^{-\frac{2 a}{u} j^{2}(a+u b)} e^{\frac{2 a}{u} j y} .
\end{aligned}
$$

Proof of Proposition 3.1. To simplify notation we suppose that $t=0$. Let $x, y \in \mathbb{R}$ and $u \in(0, \infty)$.

Note that

$$
\begin{aligned}
P\left(\rho_{u}<\rho_{l}\right) & =\int_{0}^{T} P_{x}\left(W_{t}>b t \text { for all } t \in[0, v] \mid \rho_{u}=v\right) P\left(\rho_{u} \in d v\right) \\
& =\int_{0}^{T} P_{x, v, b T}\left(W_{t}>b t, \forall t \in[0, v] \mid W_{t}<b T, \forall t \in[0, v)\right) f(b T-x, v) d v .
\end{aligned}
$$

Since a time-reversed Brownian bridge is again a Brownian bridge (see, e.g., Proposition 1 in [21]) we have

$$
\begin{aligned}
& P_{x, v, b T}\left(W_{t}>b t, W_{t}<b T, \text { for all } t \in[0, v)\right) \\
& =P_{0, v, x-b T}\left(W_{t}>b v-b T-b t, W_{t}<0, \text { for all } t \in[0, v)\right) \\
& =P_{0, v, b T-x}\left(W_{t}<b T+b t-b v, W_{t}>0, \text { for all } t \in[0, v)\right)
\end{aligned}
$$

and

$$
P_{x, v, b T}\left(W_{t}<b T \text {, for all } t \in[0, v)\right)=P_{0, v, b T-x}\left(W_{t}>0 \text {, for all } t \in[0, v)\right) .
$$

This further implies

$$
\begin{aligned}
& P_{x, v, b T}\left(W_{t}>b t, \forall t \in[0, v] \mid W_{t}<b T, \forall t \in[0, v)\right) \\
& =P_{0, v, b T-x}\left(W_{t}<b T-b v+b t \text { for all } t \in[0, v] \mid W_{t}>0, \forall t \in[0, v)\right) .
\end{aligned}
$$

By Lemma 3.2 the conditional probability in the last line of the previous equation is equal to $g(b T-x, v, b(T-v), b)$. Combining this with (3.4) yields the claim.

With the analytic formula (3.2) we can show that $H$ solves the heat equation in the interior of $J$. More precisely, we have the following.

Theorem 3.3. We have $H \in \mathcal{C}(\bar{J} \backslash\{(T, T)\}) \cap \mathcal{C}^{1,2}(J)$. Moreover, $H$ satisfies the heat equation on $J$ with the boundary conditions

$$
H(t, b t)=1 \text { and } H(t, b T)=0,
$$

for all $t \in[0, T)$. 
Proof of Theorem 3.3. With the analytic representation (3.2) at hand one can show, with some tedious but elementary calculations, that $H$ satisfies the claimed continuity and smoothness. Since the calculations are rather long, we present them in the Appendix A.

Now, one can show that $H$ satisfies the heat equation on $J$ by using Ito's formula. To do so, choose an arbitrary point $(t, x) \in J$ and let $B^{h}$ be the ball of radius $h>0$ around $(t, x)$. Stop the Brownian motion $W^{t, x}$ when the pair $\left(s, W_{s}^{t, x}\right)_{s \geq t}$ hits the boundary of $B^{h}$. Then apply Ito's formula on $H\left(s, W_{s}^{t, x}\right)$ between $t$ and the boundary hitting time. Since $H\left(s, W_{s}^{t, x}\right)$ is a martingale, the bounded variation part of the Ito process decomposition vanishes. By using the mean value theorem and letting $h \downarrow 0$ one can show that the heat equation is satisfied at $(t, x)$. The boundary conditions follow from the definition of $H$.

\section{Solving the control problem}

In this section we prove Theorem 2.1. To simplify notation we assume, throughout the section, that $\gamma=1$.

We first derive an alternative representation of the value function. For all $(t, x) \in$ $[0, T] \times[0, \infty)$ and $\alpha \in \mathcal{A}$ we define

$$
\begin{aligned}
\tau_{l}^{t, x, \alpha} & =\inf \left\{s \geqslant t: X_{s}^{t, x, \alpha} \leqslant 0\right\}, \\
\tau_{u}^{t, x, \alpha} & =\inf \left\{s \geqslant t: X_{s}^{t, x, \alpha} \geqslant T-s\right\} .
\end{aligned}
$$

Moreover, we set $\widehat{\alpha}_{s}^{t, x}=\alpha_{s} 1_{\left\{\tau_{u}^{t, x, \alpha}>s\right\}}$ for all $s \in[t, T]$. Notice that

$$
J(t, x, \alpha) \geqslant J\left(t, x, \widehat{\alpha}^{t, x}\right)=P\left(\tau_{l}^{t, x, \alpha}<\tau_{u}^{t, x, \alpha}\right) .
$$

Therefore, the value function satisfies

$$
V(t, x)=\inf \left\{P\left(\tau_{l}^{t, x, \alpha}<\tau_{u}^{t, x, \alpha}\right): \alpha \in \mathcal{A}\right\} .
$$

We are going to prove that the value function $V$ is a classical solution of the following HJB equation:

$$
\partial_{t} V(t, x)-\partial_{x} V(t, x)+\inf _{a \in[0,1]} \frac{1}{2} a^{2} \partial_{x x} V(t, x)=0,
$$

with the boundary conditions

$$
\begin{aligned}
V(t, 0) & =1, \text { for all } t \in[0, T), \\
V(t, T-t) & =0, \text { for all } t \in[0, T] .
\end{aligned}
$$

To do so, we first construct an explicit solution of the HJB equation (4.2).

Let $I=\{(t, x) \in[0, T) \times(0, T): x<T-t\}$. We denote by $\bar{I}$ the closure of $I$, i.e. $\bar{I}=\{(t, x) \in[0, T] \times[0, T]: x \leqslant T-t\}$. Recall the definition of $Y^{t, x}$ given in Theorem 2.1: $Y^{t, x}$ is the solution of the $\operatorname{SDE} d Y_{s}=-d s+a^{\star}\left(s, Y_{s}\right) d W_{s}$ on $[t, T]$ with initial condition $x$, where $a^{\star}(t, x)=1$ if $(t, x) \in I$ and 0 otherwise.

For all $(t, x) \in \bar{I}$ let

$$
\begin{aligned}
\sigma_{u}^{t, x} & =\inf \left\{s \geqslant t: Y_{s}^{t, x} \geqslant T-s\right\} \\
\sigma_{l}^{t, x} & =\inf \left\{s \geqslant t: Y_{s}^{t, x} \leqslant 0\right\} .
\end{aligned}
$$

and

$$
G(t, x):=P\left(\sigma_{l}^{t, x}<\sigma_{u}^{t, x}\right)
$$


Lemma 4.1. We have $G \in \mathcal{C}(\bar{I} \backslash\{(T, 0)\}) \cap \mathcal{C}^{1,2}(I)$. Moreover, for all $(t, x) \in I$

$$
\partial_{t} G(t, x)-\partial_{x} G(t, x)+\frac{1}{2} \partial_{x x} G(t, x)=0 .
$$

and $\partial_{x x} G(t, x) \leqslant 0$.

Proof. Notice that $G(t, x)=H(t, x+t)$ for all $(t, x) \in \bar{I}$, where $H$ is the function introduced in (3.1) with $b=1$. Hence the smoothness of $G$ on $I$ and the continuity on $\bar{I} \backslash\{(T, 0)\}$ follow from the smoothness and continuity of $H$ on $J$ and $\bar{J}$, respectively.

In addition, observe that for all $(t, x) \in J$ we have

$$
\partial_{t} H(t, x)=\partial_{t} G(t, x-t)-\partial_{x} G(t, x-t) \quad \text { and } \quad \partial_{x x} H(t, x)=\partial_{x x} G(t, x-t) .
$$

Since $H$ satisfies the heat equation, this implies that (4.3) is satisfied for all $(t, x) \in$ $I$.

Finally notice that, because of the particular triangular geometry of the problem, for fixed $x$, if we consider an initial time $t^{\prime}>t$, then the process starts closer to the hypothenuse, which implies that $\partial_{t} H \geqslant 0$. Hence, with the heat equation, we obtain $\frac{1}{2} \partial_{x x} H(t, x)=-\partial_{t} H(t, x) \leqslant 0$. This entails $\partial_{x x} G(t, x) \leqslant 0$ on $I$.

Lemma 4.1 implies that $G$ is concave in $x$ and that $G$ satisfies the HJB equation (4.2).

Lemma 4.2. $G$ is a solution of the HJB equation (4.2) on I, and satisfies the boundary conditions

$$
G(t, T-t)=0 \text { for } t \in[0, T] \text {, and } G(t, 0)=1 \text { for } t \in[0, T) .
$$

Proof. Lemma 4.1 implies $\partial_{x x} G(t, x)=\inf _{a \in[0,1]} a^{2} \partial_{x x} G(t, x)$, and hence $G$ satisfies the HJB equation (4.2) on $I$.

The boundary properties (4.4) follow directly from the definition of $G$.

Proof of Theorem 2.1. It is enough to prove that $G(t, x)=V(t, x)$ for all $(t, x) \in \bar{I}$.

First observe that $V(t, T-t)=0$ for all $t \in[0, T]$ and $V(t, 0)=1$ for all $t \in[0, T)$. Hence $V$ and $G$ coincide on the boundary of $\bar{I}$.

Now let $(t, x) \in I$. From the definition of $G$ and formula (4.1) we have $G(t, x) \geqslant$ $V(t, x)$. In order to prove $G(t, x) \leqslant V(t, x)$, let $\alpha$ be an arbitrary control in $\mathcal{A}$. Let $\tau_{n}=\inf \left\{s \geqslant t: d\left(\left(s, X_{s}^{t, x, \alpha}\right), \partial I\right) \leqslant \frac{1}{n}\right\}$, where $\partial I=\bar{I} \backslash I$ denotes the boundary of $I$ and $d(\cdot, \partial I)$ the distance to the boundary (here we set $\inf \emptyset=t)$. The Ito formula yields

$$
\begin{aligned}
G\left(\tau_{n}, X_{\tau_{n}}^{t, x, \alpha}\right)= & G(t, x)+\int_{t}^{\tau_{n}} \partial_{x} G\left(s, X_{s}^{t, x, \alpha}\right) d W_{s} \\
& +\int_{t}^{\tau_{n}}\left[\partial_{t} G\left(s, X_{s}^{t, x, \alpha}\right)-\partial_{x} G\left(s, X_{s}^{t, x, \alpha}\right)+\frac{1}{2} \alpha_{s}^{2} \partial_{x x} G\left(s, X_{s}^{t, x, \alpha}\right)\right] d s .
\end{aligned}
$$

Since $G$ satisfies the HJB equation (4.2) on $I$ we further obtain

$$
G\left(\tau_{n}, X_{\tau_{n}}^{t, x, \alpha}\right) \geqslant G(t, x)+\int_{t}^{\tau_{n}} \partial_{x} G\left(s, X_{s}^{t, x, \alpha}\right) d W_{s} .
$$

Note that the integrand of the stochastic integral in (4.5) is bounded and hence the expectation of the stochastic integral vanishes. We thus obtain

$$
E\left[G\left(\tau_{n}, X_{\tau_{n}}^{t, x, \alpha}\right)\right] \geqslant G(t, x) .
$$

Since $G$ is bounded and continuous in $\bar{I} \backslash\{(T, 0)\}$, dominated convergence implies $J(t, x, \alpha) \geqslant J\left(t, x, \alpha^{t, x}\right)=E\left[G\left(\tau, X_{\tau}^{t, x, \alpha}\right)\right] \geqslant G(t, x)$, where $\tau$ is the first time at which $X^{t, x, \alpha}$ hits the boundary $\partial I$. Therefore, $V(t, x) \geqslant G(t, x)$. 


\section{A. Appendix}

In this appendix we prove the continuity and smoothness properties of the function $H$ claimed in Theorem 3.3. To shorten notation, we take $b=1$ and we show the respective properties for

$$
\bar{H}(t, x):=1-H(t, x)=P\left(\rho_{u}^{t, x}<\rho_{\ell}^{t, x}\right) .
$$

Lemma A.1. For every $t, x \in(t, T) \mapsto \bar{H}(t, x)$ is continuous.

Proof. Let $t \in[0, T)$ and $\delta>0$, we prove that for all $x \in[t, T-\delta]$, the function is continuous in $x$. For $t<x<T-\delta$, using Proposition 3.1, and a simple change of variables, we get

$$
\bar{H}(t, x)=\int_{0}^{T-t} \frac{1}{\sqrt{2 \pi} u^{3 / 2}} \exp \left(-\frac{(T-x)^{2}}{2 u}\right) \sum_{k=-\infty}^{+\infty} h_{k}(u, x) d u
$$

with

$$
\begin{aligned}
h_{k}(u, x):=[T- & x-2 k(T-t)] \\
& \times \exp \left(-2 k^{2} \frac{(T-u-t)(T-t)}{u}\right) \exp \left(2 k \frac{(T-u-t)(T-x)}{u}\right) .
\end{aligned}
$$

Note that $\sum_{k=-\infty}^{+\infty} h_{k}(u, x) \leqslant 1$, since it is a probability.

1. For all $x \in[t, T-\delta]$,

$$
\frac{1}{\sqrt{2 \pi} u^{3 / 2}} \exp \left(-\frac{(T-x)^{2}}{2 u}\right) \leq \frac{1}{\sqrt{2 \pi} u^{3 / 2}} \exp \left(-\frac{(T-(T-\delta))^{2}}{2 u}\right)
$$

and this function $u \mapsto \frac{1}{\sqrt{2 \pi} u^{3 / 2}} \exp \left(-\frac{(T-(T-\delta))^{2}}{2 u}\right)$ is integrable on $(0, T-t)$.

2. $x \mapsto \frac{1}{\sqrt{2 \pi} u^{3 / 2}} \exp \left(-\frac{(T-x)^{2}}{2 u}\right)$ is continuous on $[t, T-\delta]$.

3. Let us prove that the series $\sum_{k=-\infty}^{+\infty} h_{k}(u, x)$ is a continuous function of $x$. Since each $h_{k}$ is a continuous function of $x$, it is sufficient to prove that the series converges uniformly, which is implied by the fact that

$$
\begin{aligned}
& \sum_{k=-\infty}^{+\infty} \sup _{t \leq x \leq T}\left|h_{k}(u, x)\right|<+\infty, \text { since } \\
& \left|h_{k}(u, x)\right| \leq(T-t)|2 k-1| \exp \left(-2 \frac{(T-u-t)(T-t)}{u} k(k-1)\right) .
\end{aligned}
$$

The point 1 . above and the fact that $\sum_{k=-\infty}^{+\infty} h_{k}(u, x) \leqslant 1$ show that the integrand in (A.1) is bounded from above by an integrable function, which is independent of $x$. The points 2. and 3. allow to deduce that the integrand in (A.1) is a continuous function of $x$. Hence $x \mapsto \bar{H}(t, x)$ is continuous, for $t<x<T$.

Let us now prove that $\lim _{x \rightarrow T^{-}} \bar{H}(t, x)=1$. Recall that

$$
\begin{aligned}
\bar{H}(t, x)=\int_{t}^{T} f(u) & \sum_{k=-\infty}^{+\infty}\left(1-2 k \frac{T-t}{T-x}\right) \\
& \times \exp \left(\frac{T-u}{u-t}\left[-2 k^{2}(T-t)+2 k(T-x)\right]\right) d u
\end{aligned}
$$


with

$$
f(u)= \begin{cases}\frac{T-x}{\sqrt{2 \pi}(u-t)^{3 / 2}} \exp \left(-\frac{(T-x)^{2}}{2(u-t)}\right) & \text { if } x<T \\ \mathbf{1}_{\{u=t\}} & \text { if } x=T .\end{cases}
$$

Isolating the term $k=0$ in the sum, we get,

$$
\begin{aligned}
\bar{H}(t, x)= & \int_{t}^{T}\left\{f(u)+\frac{1}{\sqrt{2 \pi}(u-t)^{3 / 2}} \exp \left(-\frac{(T-x)^{2}}{2(u-t)}\right)\right. \\
& \left.\times \sum_{k=-\infty, k \neq 0}^{+\infty}(T-x-2 k(T-t)) \exp \left(\frac{T-u}{u-t}\left[-2 k^{2}(T-t)+2 k(T-x)\right]\right)\right\} d u .
\end{aligned}
$$

When $x \rightarrow T$, the second term in the previous integral simplifies to

$$
\frac{1}{\sqrt{2 \pi}(u-t)^{3 / 2}} \sum_{k=-\infty, k \neq 0}^{+\infty}(-2 k(T-t)) \exp \left(\frac{T-u}{u-t}\left[-2 k^{2}(T-t)\right]\right)=0,
$$

and

$$
\lim _{x \rightarrow T} \bar{H}(t, x)=\lim _{x \rightarrow T} \int_{t}^{T} f(u) d u=1 .
$$

Finally, for $x=t$, we have

$$
\bar{H}(t, t)=\int_{t}^{T} f(u) \sum_{k=-\infty}^{+\infty}(1-2 k) \exp \left(\frac{(T-u)(T-t)}{u-t} 2 k(1-k)\right) d u=0,
$$

since

$$
\sum_{k=-N}^{N}(1-2 k) \exp (C k(1-k))=\frac{2 N+1}{\exp (C N(N+1))}
$$

goes to 0 as $N$ goes to $+\infty$, for any $C>0$.

The following observations will be useful: we can write $\bar{H}(t, x)$ in the following way:

$$
\begin{aligned}
\bar{H}(t, x)= & \int_{t}^{T} \frac{1}{\sqrt{2 \pi}(u-t)^{3 / 2}} \exp \left(-\frac{(T-x)^{2}}{2(u-t)}\right) \\
& \times\left\{T-x-4(T-t) \sum_{k=1}^{+\infty} k \exp \left(-2 k^{2} C_{t, u, T}\right) \sinh \left(2 k(T-x) \frac{T-u}{u-t}\right)\right. \\
& \left.+2(T-x) \sum_{k=1}^{+\infty} \exp \left(-2 k^{2} C_{t, u, T}\right) \cosh \left(2 k(T-x) \frac{T-u}{u-t}\right)\right\} d u,
\end{aligned}
$$

with $C_{t, u T}:=\frac{(T-t)(T-u)}{u-t}$. This can be written

$$
\bar{H}(t, x)=\int_{t}^{T} h(x, u) d u
$$

with

$$
h(x, u):=g(x, u) \times\left\{T-x-4(T-t) \sum_{k=1}^{+\infty} f_{k}^{1}(x, u)+2(T-x) \sum_{k=1}^{+\infty} f_{k}^{2}(x, u)\right\} .
$$


Lemma A.2. Both series $\sum f_{k}^{1}$ and $\sum f_{k}^{2}$ are $\mathcal{C}^{2}$ functions of $x$ and

$$
\frac{\partial^{2}}{\partial x^{2}} \sum f_{k}^{i}=\sum \frac{\partial^{2}}{\partial x^{2}} f_{k}^{i}, \quad i=1,2
$$

Proof. We will prove the claim for $\sum f_{k}^{1}$, the proof for $\sum f_{k}^{2}$ being very similar. For any $k \geq 1$, the map $x \mapsto f_{k}^{1}(x, u)$ is $C^{2}$ and

$$
\frac{\partial^{2}}{\partial x^{2}} f_{k}^{1}(x, u)=4 k^{3} \exp \left(-2 k^{2} C_{t, u, T}\right)\left(\frac{T-u}{u-t}\right)^{2} \sinh \left(2 k(T-x) \frac{T-u}{u-t}\right) .
$$

The function sinh being increasing,

$$
\left|\frac{\partial^{2}}{\partial x^{2}} f_{k}^{1}(x, u)\right| \leq 4 k^{3} \exp \left(-2 k^{2} C_{t, u, T}\right)\left(\frac{T-u}{u-t}\right)^{2} \sinh \left(2 k(T-t) \frac{T-u}{u-t}\right),
$$

which implies that the series $\sum \frac{\partial^{2}}{\partial x^{2}} f_{k}^{1}(x, u)$ converges uniformy, and from which we deduce the claim.

Lemma A.3. For every $t, x \mapsto \bar{H}(t, x)$ is $\mathcal{C}^{2}$.

Proof. As previously, let $x \in[t, T-\delta]$ where $\delta>0$. We have

$$
\begin{aligned}
\frac{\partial g}{\partial x}(x, u) & =\frac{1}{\sqrt{2 \pi}(u-t)^{3 / 2}} \exp \left(-\frac{(T-x)^{2}}{2(u-t)}\right)\left[\frac{T-x}{u-t}\right], \\
\frac{\partial^{2} g}{\partial x^{2}}(x, u) & =\frac{1}{\sqrt{2 \pi}(u-t)^{3 / 2}} \exp \left(-\frac{(T-x)^{2}}{2(u-t)}\right)\left[\frac{(T-x)^{2}}{(u-t)^{2}}-\frac{1}{u-t}\right] .
\end{aligned}
$$

Using Lemma A.2, we get

$$
\frac{\partial^{2} h}{\partial x^{2}}(x, u)=\frac{1}{\sqrt{2 \pi}(u-t)^{3 / 2}} \exp \left(-\frac{(T-x)^{2}}{2(u-t)}\right)\left(I_{1}+I_{2}+I_{3}\right)
$$

where

$$
\begin{aligned}
I_{1}:=\left[\frac{(T-x)^{2}}{(u-t)^{2}}-\frac{1}{u-t}\right]\left\{T-x-4(T-t) \sum_{k=1}^{+\infty} f_{k}^{1}(x, u)+2(T-x) \sum_{k=1}^{+\infty} f_{k}^{2}(x, u)\right\}, \\
I_{2}:=-16(T-t) \frac{(T-u)^{2}}{(u-t)^{2}} \sum_{k=1}^{+\infty} k^{3} \exp \left(-2 k^{2} C_{t, u, T}\right) \sinh \left(2 k(T-x) \frac{T-u}{u-t}\right) \\
\quad+8 \frac{T-u}{u-t} \sum_{k=1}^{+\infty} k \exp \left(-2 k^{2} C_{t, u, T}\right) \sinh \left(2 k(T-x) \frac{T-u}{u-t}\right) \\
\quad+8(T-x) \frac{(T-u)^{2}}{(u-t)^{2}} \sum_{k=1}^{+\infty} k^{2} \exp \left(-2 k^{2} C_{t, u, T}\right) \cosh \left(2 k(T-x) \frac{T-u}{u-t}\right),
\end{aligned}
$$


and

$$
\begin{aligned}
I_{3}:= & {\left[\frac{T-x}{u-t}\right] \times } \\
\{ & -1+8 C_{t, u, T} \sum_{k=1}^{+\infty} k^{2} \exp \left(-2 k^{2} C_{t, u, T}\right) \cosh \left(2 k(T-x) \frac{T-u}{u-t}\right) \\
& -2 \sum_{k=1}^{+\infty} \exp \left(-2 k^{2} C_{t, u, T}\right) \cosh \left(2 k(T-x) \frac{T-u}{u-t}\right) \\
& \left.-4 \frac{(T-u)(T-x)}{u-t} \sum_{k=1}^{+\infty} k \exp \left(-2 k^{2} C_{t, u, T}\right) \sinh \left(2 k(T-x) \frac{T-u}{u-t}\right)\right\} .
\end{aligned}
$$

We have

$$
\left|\frac{1}{\sqrt{2 \pi}(u-t)^{3 / 2}} \exp \left(-\frac{(T-x)^{2}}{2(u-t)}\right) I_{1}\right| \leq g(x, u)\left(\frac{T-x}{u-t}\right)^{2}+g(x, u) \frac{1}{u-t} .
$$

For any $x \in[t, T-\delta]$, we have

$$
g(x, u)\left(\frac{T-x}{u-t}\right)^{2} \leq \frac{(T-t)^{2}}{\sqrt{2 \pi}(u-t)^{5 / 2}} \exp \left(-\frac{\left(T-(T-\delta)^{2}\right.}{2(u-t)}\right)
$$

and

$$
g(x, u) \frac{1}{u-t} \leq \frac{1}{\sqrt{2 \pi}(u-t)^{5 / 2}} \exp \left(-\frac{\left(T-(T-\delta)^{2}\right.}{2(u-t)}\right)
$$

The two functions on the RHS are integrable on $(0, T-t)$.

We can show that all the terms appearing in the quantities $\left|\frac{1}{\sqrt{2 \pi}(u-t)^{3 / 2}} \exp \left(-\frac{(T-x)^{2}}{2(u-t)}\right) I_{2}\right|$ and $\left|\frac{1}{\sqrt{2 \pi}(u-t)^{3 / 2}} \exp \left(-\frac{(T-x)^{2}}{2(u-t)}\right) I_{3}\right|$ are bounded from above by $\leq \frac{K}{(u-t)^{1 / 2}}$, for $u \in$ $(t, t+\varepsilon)$, where $\varepsilon>0$ and where $K$ is a positive constant (independent of $u$ and $x$ ).

Let us for example study in detail the term

$$
\begin{aligned}
\tilde{I}_{2}:=\frac{1}{\sqrt{2 \pi}(u-t)^{3 / 2}} & \exp \left(-\frac{(T-x)^{2}}{2(u-t)}\right) 16(T-t) \frac{(T-u)^{2}}{(u-t)^{2}} \\
& \times \sum_{k=1}^{+\infty} k^{3} \exp \left(-2 k^{2} C_{t, u, T}\right) \sinh \left(2 k(T-x) \frac{T-u}{u-t}\right)
\end{aligned}
$$

the arguments for the other terms are very similar.

Using that sinh is increasing and that $C_{t, u, T}=\frac{(T-t)^{2}}{u-t}-(T-t)$, we get

$$
\begin{aligned}
\left|\tilde{I}_{2}\right| \leq & \frac{1}{\sqrt{2 \pi}(u-t)^{3 / 2}} \exp \left(-\frac{(T-x)^{2}}{2(u-t)}\right) 16 \frac{(T-t)^{3}}{(u-t)^{2}} \\
& \times \sum_{k=1}^{+\infty} k^{3} \exp \left(-k^{2}\left[\frac{2(T-t)^{2}}{u-t}-2(T-t)\right]\right) \sinh \left(2 k \frac{(T-t)^{2}}{u-t}\right) .
\end{aligned}
$$

Define

$$
S(u):=\sum_{k=1}^{+\infty} k^{3} \exp \left(-k^{2}\left[\frac{c}{u}-c^{\prime}\right]\right) \sinh \left(k \frac{c}{u}\right), \quad 0<u \leq(T-t)
$$


where $c=2(T-t)^{2}$ and $c^{\prime}=2(T-t)\left(\frac{c}{u}-c^{\prime} \geq 0\right.$ for $\left.u \leq T-t\right)$. We are going to prove that $\lim _{u \rightarrow 0^{+}} u \log S(u)=0$. Assuming momentarily that this limit is indeed equal to 0 , then there exists $\bar{u}>t$ such that for any $u \leq \bar{u}$,

$$
2(u-t) \log S(u-t)-2(u-t) \log \left(\frac{(2 \pi)^{1 / 6}(u-t)}{2^{4 / 3}(T-t)}\right)^{3} \leq T-(T-\delta) .
$$

This implies for any $x \in[t, T-\delta]$ and any $u \leq \bar{u}$,

$$
\frac{1}{\sqrt{2 \pi}(u-t)^{3 / 2}} \exp \left(-\frac{(T-x)^{2}}{2(u-t)}\right) 16 \frac{(T-t)^{3}}{(u-t)^{2}} S(u-t) \leq \frac{1}{(u-t)^{1 / 2}} .
$$

This implies, by splitting the interval $[t, T]$ into $[t, t+\varepsilon)$ and $[t+\varepsilon, T]$ with $\varepsilon$ small enough, that $\left|\frac{\partial^{2} h}{\partial x^{2}}(x, u)\right|$ is bounded from above by an integrable function which is independent of $x$, on each of these intervals. Using similar arguments as in the proof of Lemma A.2, we can show that $x \mapsto \frac{\partial^{2} h}{\partial x^{2}}(x, u)$ is a continuous function. From this, we can conclude that $x \mapsto \bar{H}(t, x)$ is $\mathcal{C}^{2}$.

It remains to prove that $\lim _{u \rightarrow 0^{+}} u \log S(u)=0$, which is the purpose of Lemma A.4.

Lemma A.4. $\lim _{u \rightarrow 0^{+}} u \log S(u)=0$.

Proof. Define,

$$
\varphi(v, u):=\frac{1}{2} v^{3} \exp \left(-v^{2}\left[\frac{c}{u}-c^{\prime}\right]\right)\left(\exp \left(v \frac{c}{u}\right)-\exp \left(-v \frac{c}{u}\right)\right), \quad v>1,
$$

which is a differentiable function such that

$$
\frac{\partial \varphi}{\partial v}(v, u)=\exp \left(-v^{2}\left[\frac{c}{u}-c^{\prime}\right]\right) v^{2}\left[v \frac{c}{u} \cosh \left(v \frac{c}{u}\right)-\left(2 v^{2}\left[\frac{c}{u}-c^{\prime}\right]-3\right) \sinh \left(v \frac{c}{u}\right)\right] .
$$

The sign of this derivative is given by the sign of $v \frac{c}{u} \cosh \left(v \frac{c}{u}\right)-\left(2 v^{2}\left[\frac{c}{u}-c^{\prime}\right]-3\right) \sinh \left(v \frac{c}{u}\right)$, which is going to be negative for $u$ small enough. Indeed, this quantity is negative if and only if

$$
\tanh \left(v \frac{c}{u}\right)\left(2 v-2 v \frac{c^{\prime}}{c} u-\frac{3}{v} \frac{u}{c}\right)>1
$$

Since $\tanh \left(v \frac{c}{u}\right)\left(2 v-2 v \frac{c^{\prime}}{c} u-\frac{3}{v} \frac{u}{c}\right)$ converges to $2 v>1$ as $u$ goes to 0 , we have that $v \mapsto \varphi(v, u)$ is decreasing for $u$ small enough. We can then apply the standard technique of comparison between series and integrals: for $k \geq 1$,

$$
\int_{k}^{k+1} \varphi(v, u) d v \leq \varphi(k, u)=\int_{k-1}^{k} \varphi(k, u) d v \leq \int_{k-1}^{k} \varphi(v, u) d v .
$$

Taking the sum from $k=2$ to $k=N$, and then the limit when $N$ goes to $+\infty$, we have

$$
\varphi(1, u)+\int_{2}^{+\infty} \varphi(v, u) d v \leq \sum_{k=1}^{+\infty} \varphi(k, u) \leq \varphi(1, u)+\int_{1}^{+\infty} \varphi(v, u) d v .
$$


Let us compute the integral on the right hand side, (which is well defined since for $v$ in the neighbourhood of $+\infty, \varphi(v, u)=o\left(\frac{1}{v^{p}}\right)$ for any $\left.p>1\right)$, that we expand as:

$$
\begin{aligned}
K & :=\int_{1}^{+\infty} \varphi(v, u) d v=\frac{1}{2}\left(K^{+}-K^{-}\right), \quad \text { where } \\
K^{+} & :=\int_{1}^{+\infty} v^{3} \exp \left(-v^{2}\left[\frac{c}{u}-c^{\prime}\right]+v \frac{c}{u}\right) d v \quad \text { and } \\
K^{-} & :=\int_{1}^{+\infty} v^{3} \exp \left(-v^{2}\left[\frac{c}{u}-c^{\prime}\right]-v \frac{c}{u}\right) d v .
\end{aligned}
$$

Writing $\bar{\Phi}_{j}(x):=E\left[Z^{j} \mathbf{1}_{\{Z>x\}}\right], j \geq 0$ and $\bar{\Phi}(x):=\bar{\Phi}_{0}(x)=P(Z>x)$, with $Z$ a r.v. having a standard Gaussian distribution, we have

$$
\begin{aligned}
K^{+} & =\sqrt{2 \pi} \frac{\exp \left(\frac{c^{2}}{4 u^{2}\left[\frac{c}{u}-c^{\prime}\right]}\right)}{4\left[\frac{c}{u}-c^{\prime}\right]^{2}} \times \\
& {\left[\kappa_{2}^{3} \bar{\Phi}\left(\kappa_{1}-\kappa_{2}\right)+3 \kappa_{2}^{2} \bar{\Phi}_{1}\left(\kappa_{1}-\kappa_{2}\right)+3 \kappa_{2} \bar{\Phi}_{2}\left(\kappa_{1}-\kappa_{2}\right)+\bar{\Phi}_{3}\left(\kappa_{1}-\kappa_{2}\right)\right], }
\end{aligned}
$$

where $\kappa_{1}(u):=\sqrt{2\left(\frac{c}{u}-c^{\prime}\right)}$ and $\kappa_{2}(u):=\frac{c}{u \kappa_{1}(u)}$ (for simplicity, we do not indicate the dependence on $u$ of $\kappa_{1}$ and $\kappa_{2}$ when there is no ambiguity). Similarly we have

$$
\begin{aligned}
K^{-}=\sqrt{2 \pi} \frac{\exp \left(\frac{c^{2}}{4 u^{2}\left[\frac{c}{u}-c^{\prime}\right]}\right)}{4\left[\frac{c}{u}-c^{\prime}\right]^{2}} \times \\
\quad\left[\bar{\Phi}_{3}\left(\kappa_{1}+\kappa_{2}\right)-3 \kappa_{2} \bar{\Phi}_{2}\left(\kappa_{1}+\kappa_{2}\right)+3 \kappa_{2}^{2} \bar{\Phi}_{1}\left(\kappa_{1}+\kappa_{2}\right)-\kappa_{2}^{3} \bar{\Phi}\left(\kappa_{1}+\kappa_{2}\right)\right] .
\end{aligned}
$$

By an integration by parts,

$$
\begin{aligned}
\bar{\Phi}_{3}(x) & =\frac{1}{\sqrt{2 \pi}} \exp \left(-\frac{x^{2}}{2}\right)\left(x^{2}+2\right) \\
\bar{\Phi}_{2}(x) & =\frac{x}{\sqrt{2 \pi}} \exp \left(-\frac{x^{2}}{2}\right)+\bar{\Phi}(x) \\
\bar{\Phi}_{1}(x) & =\frac{1}{\sqrt{2 \pi}} \exp \left(-\frac{x^{2}}{2}\right), \quad \text { and it is standard that } \\
\bar{\Phi}(x) & \sim \frac{1}{x} \frac{1}{\sqrt{2 \pi}} \exp \left(-\frac{x^{2}}{2}\right) .
\end{aligned}
$$

Using these explicit expressions, the value of $\varphi(1, u)$ given through (A.3) and inequality (A.4), it is straightforward to show that $u \log S(u)$ converge to 0 as $u$ goes to $0^{+}$.

Lemma A.5. For every $x \in(0, T), t \in[0, x] \mapsto \bar{H}(t, x)$ is $\mathcal{C}^{1}$.

Proof. Recall that (do a change of variable $u=\frac{u-t}{T-t}$ )

$$
\begin{aligned}
\bar{H}(t, x)= & \int_{0}^{T}\left\{f(u, t, x)+\frac{1}{\sqrt{2 \pi}(T-t)^{1 / 2}(u)^{3 / 2}} \exp \left(-\frac{(T-x)^{2}}{2(T-t) u}\right)\right. \\
& \left.\times \sum_{k=-\infty, k \neq 0}^{+\infty}(T-x-2 k(T-t)) \exp \left(\frac{1-u}{u}\left[-2 k^{2}(T-t)+2 k(T-x)\right]\right)\right\} d u . \\
= & \int_{0}^{T}\left\{f(u, t, x)+g(t, x, u) \sum_{k=-\infty, k \neq 0}^{+\infty} h_{k}(t, x, u)\right\} d u,
\end{aligned}
$$


where

$$
f(u, t, x)= \begin{cases}\frac{T-x}{\sqrt{2 \pi}(T-t)^{1 / 2}(u)^{3 / 2}} \exp \left(-\frac{(T-x)^{2}}{2(T-t) u}\right) & \text { if } x<T \\ \mathbf{1}_{\{u=t\}} & \text { if } x=T .\end{cases}
$$

Let $x \in[0, T), t_{0} \leq 0$ and $\delta>0$ such that $t_{0}+\delta \in[0, x)$.

- For all $t \in\left[0, t_{0}+\delta\right]$

$$
\begin{aligned}
\left|\frac{\partial f}{\partial t}\right|= & \frac{T-x}{2 \sqrt{2 \pi}(T-t)^{3 / 2}(u)^{3 / 2}} \exp \left(-\frac{(T-x)^{2}}{2(T-t) u}\right) \\
& +\frac{(T-x)^{3}}{\sqrt{2 \pi}(T-t)^{3 / 2}(u)^{5 / 2}} \exp \left(-\frac{(T-x)^{2}}{2(T-t) u}\right) \\
\leq & \frac{T-x}{2 \sqrt{2 \pi}\left(T-t_{0}-\delta\right)^{3 / 2}(u)^{3 / 2}} \exp \left(-\frac{(T-x)^{2}}{2 T u}\right) \\
& +\frac{(T-x)^{3}}{\sqrt{2 \pi}\left(T-t_{0}-\delta\right)^{3 / 2}(u)^{5 / 2}} \exp \left(-\frac{(T-x)^{2}}{2 T u}\right) .
\end{aligned}
$$

The right side is a integrable function on $[0,1]$ independent of $t \in\left[0, t_{0}+\delta\right]$. The function $t \mapsto \frac{\partial f}{\partial t}$ is continuous on $\left[0, t_{0}+\delta\right]$

- Note that $g(t, x, u)=f(t, x, u) /(T-x)$. Hence the functions $t \mapsto g(t, x, u)$ and $t \mapsto \frac{\partial g}{\partial t}$ are continuous on $\left[0, t_{0}+\delta\right]$ and can be bounded by above by a function independent of $f$ and integrable w.r.t $u$.

- Let us prove that the $\sum_{k=-\infty, k \neq 0}^{+\infty} h_{k}(t, x, u)$ is a $\mathcal{C}^{1}$ function. Since

$$
\begin{aligned}
& \sup _{t \in\left[0, t_{0}+\delta\right]}\left|\frac{\partial h_{k}(t, x, u)}{\partial t}\right| \\
= & \sup _{t \in\left[0, t_{0}+\delta\right]} \mid 2 k \exp \left(\frac{1-u}{u}\left[-2 k^{2}(T-t)+2 k(T-x)\right]\right) \\
& +2 k^{2} \frac{1-u}{u}(T-x-2 k(T-t)) \exp \left(\frac{1-u}{u}\left[-2 k^{2}(T-t)+2 k(T-x)\right]\right) \mid \\
\leq & 2|k| \exp \left(\frac{1-u}{u}\left[-2 k^{2}\left(T-t_{0}-\delta\right)+2 k(T-x)\right]\right) \\
& +2 k^{2} \frac{1-u}{u}(T-x+2|k| T) \exp \left(\frac{1-u}{u}\left[-2 k^{2}\left(T-t_{0}-\delta\right)+2 k(T-x)\right]\right) \\
\leq & 2|k| \exp \left(\frac{1-u}{u}\left[-2 k^{2}(T-x)+2 k(T-x)\right]\right) \\
& +2 k^{2} \frac{1-u}{u}(T-x+2|k| T) \exp \left(\frac{1-u}{u}\left[-2 k^{2}(T-x)+2 k(T-x)\right]\right),
\end{aligned}
$$

which is the term of a convergent series, the result follows.

- $\left|\frac{\partial g(t, x, u)}{\partial t} \sum_{k=-\infty, k \neq 0}^{+\infty} h_{k}(t, x, u)\right| \leq\left|\frac{\partial g(t, x, u)}{\partial t}\right|$, which is bounded by above by an integrable function independent of $t \in\left[0, t_{0}+\delta\right]$ (see above).

- Define

$$
\widetilde{S}_{n}(u):=\sum_{k=1}^{+\infty} k^{n} \exp \left(-k^{2} c\left[\frac{1-u}{u}\right]\right) \sinh \left(k c \frac{1-u}{u}\right), \quad 0<u \leq(T-t),
$$


where $c=(T-x), n=1,2,3$. Similar arguments as the ones used in the proof of Lemma A.4 imply that $\lim _{u \rightarrow 0^{+}} u \log \widetilde{S}_{n}(u)=0$. Then there exists $\bar{u}$ such that for any $u \leq \bar{u}$

$$
\left|g(t, x, u) \sum_{k=-\infty, k \neq 0}^{+\infty} \frac{\partial h_{k}(t, x, u)}{\partial t}\right| \leq C \frac{1}{\sqrt{u}},
$$

where $C$ is constant independent of $u$ and $t$.

\section{References}

[1] M. Abundo. Some conditional crossing results of Brownian motion over a piecewise-linear boundary. Statist. Probab. Lett., 58(2):131-145, 2002.

[2] I. Althöfer, M. Beckmann, and F. Salzer. On some random walk games with diffusion control. In A. Plaat, J. van den Herik, and W. Kosters, editors, Advances in Computer Games, pages 65-75, Cham, 2015. Springer International Publishing.

[3] S. Ankirchner, C. Blanchet-Scalliet, and M. Jeanblanc. Controlling the occupation time of an exponential martingale. Applied Mathematics $\&$ Optimization, 76(2):415-428, Oct 2017.

[4] S. Asmussen and H. Albrecher. Ruin probabilities. World Scientific Publishing Co Pte Ltd, 2010.

[5] S. Asmussen and M. Taksar. Controlled diffusion models for optimal dividend pay-out. Insurance: Mathematics and Economics, 20(1):1 - 15, 1997.

[6] M. Avellaneda, A. Levy, and A. Parás. Pricing and hedging derivative securities in markets with uncertain volatilities. Applied Mathematical Finance, 2(2):7388, 1995.

[7] P. Azcue and N. Muler. Stochastic optimization in insurance: a dynamic programming approach. Springer, 2014.

[8] N. Bäuerle and E. Bayraktar. A note on applications of stochastic ordering to control problems in insurance and finance. Stochastics An International Journal of Probability and Stochastic Processes, 86(2):330-340, 2014.

[9] J. Bertoin, L. Chaumont, J. Pitman, et al. Path transformations of first passage bridges. Electronic Communications in Probability, 8:155-166, 2003.

[10] P. Biane and M. Yor. Quelques précisions sur le méandre brownien. Bulletin des sciences mathématiques, 112(1):101-109, 1988.

[11] A. N. Borodin and P. Salminen. Handbook of Brownian motion-facts and formulae. Probability and its Applications. Birkhäuser Verlag, Basel, second edition, 2002.

[12] S. Browne. Optimal investment policies for a firm with a random risk process: exponential utility and minimizing the probability of ruin. Mathematics of operations research, 20(4):937-958, 1995. 
[13] S. David Promislow and V. R. Young. Minimizing the probability of ruin when claims follow brownian motion with drift. North American Actuarial Journal, 9(3):110-128, 2005.

[14] L. Denis and C. Martini. A theoretical framework for the pricing of contingent claims in the presence of model uncertainty. Ann. Appl. Probab., 16(2):827-852, 052006 .

[15] A. Fokas and K. Kalimeris. The heat equation in the interior of an equilateral triangle, stud. Appl. Math, 124:283-305, 2010.

[16] C. Hipp and M. Vogt. Optimal dynamic xl reinsurance. ASTIN Bulletin: The Journal of the IAA, 33(2):193-207, 2003.

[17] Z. Liang and J. Guo. Optimal proportional reinsurance and ruin probability. Stochastic Models, 23(2):333-350, 2007.

[18] A. Matoussi, D. Possamaï, and C. Zhou. Robust utility maximization in nondominated models with 2bsde: The uncertain volatility model. Mathematical Finance, 25(2):258-287, 2015.

[19] J. M. McNamara. Optimal control of the diffusion coefficient of a simple diffusion process. Math. Oper. Res., 8(3):373-380, 1983.

[20] S. Peng. G-expectation, g-brownian motion and related stochastic calculus of itô type. In Stochastic analysis and applications, pages 541-567. Springer, 2007.

[21] P. Salminen. On last exit decompositions of linear diffusions. Studia Sci. Math. Hungar., 33(1-3):251-262, 1997.

[22] P. Salminen and M. Yor. On hitting times of affine boundaries by reflecting brownian motion and bessel processes. Periodica Mathematica Hungarica, 62(1):75-101, Mar 2011.

[23] H. Schmidli. Optimal proportional reinsurance policies in a dynamic setting. Scandinavian Actuarial Journal, 2001(1):55-68, 2001.

[24] H. Schmidli. Stochastic control in insurance. Springer Science \& Business Media, 2007.

[25] H. Schmidli et al. On minimizing the ruin probability by investment and reinsurance. The Annals of Applied Probability, 12(3):890-907, 2002.

[26] W. Smith and G. Watson. Diffusion out of a triangle. Journal of Applied Probability, 4(3):479-488, 1967.

[27] D. W. Stephens. The logic of risk-sensitive foraging preferences. Anim. Behav., 29:628-629, 1981. 